\title{
LA SYMBOLIQUE DES NOMBRES TROIS, SEPT, DOUZE ET LA VALEUR GRAPHIQUE DU ZINZOLIN DANS $L E$ TERRAIN BOUCHABALLE DE MAX JACOB ${ }^{1}$
}

\author{
Marie-Claire Durand Guiziou \\ Universidad de Las Palmas \\ mcdurand@dfm.ulpgc.es
}

\section{RÉSUMÉ}

"Astronome, astrologue, chimiste et alchimiste, mathématicien, mais de rêve, tel fut cet ani merveilleux» dira Jean Cocteau de son fidèle ami, Max Jacob. C'est cet alchimiste des mots et des nombres, des sons, des graphies et des images que nous nous proposons de découvrir ici dans une analyse sémiotique de son roman Le Terrain Bouchaballe. Notre étude porte essentiellement sur le décodage des nombres trois, sept et douze qui témoignent à la fois de l'attrait indéniable de Max Jacob pour les représentations cabalistiques et sa foi en la doctrine du Christ; elle nous confirme aussi que les bases de la théorie cubiste jacobienne -qui a influencé tant de ses contemporains— se forgeait déjà vers 1910 , lors de la conception du roman.

MOTS CLEF: symbolisme; nombres; mythologie; semiotique; esthétique cubiste.

\section{RESUMEN}

"Astrónomo, astrólogo, químico y alquimista, matemático, aunque de ensueño, ese era el amigo maravilloso» comentará Jean Cocteau acerca de su fiel amigo, Max Ja-

' C'est le Max Jacob (1876-1944) romancier qui nous intéresse ici. Mais à l'auteur du Terrain Bouchaballe s'ajoute le dramaturge - le roman fut d'abord publié sous forme de comédie-, le poète et le peintre-dessinateur, (on pourrait ajouter l'épistolier, le musicien, l'astrologue...). Homme aux multiples facettes, ce Breton né dans une famille quimpéroise juive qui a largement marqué la poésie modeme française (L'Esprit Nouveau) mais aussi le cubisme, en peinture, sera frappé par la foi et après sa conversion au catholicisme, passera des séjours en prière chez les Bénédictins de Saint-Benoît-sur-Loire, avant d'être artêté par la Gestapo.

Toutes nos références du Terrain Bouchaballe se rapportent à l'édition de Gallimard de 1964. 
cob. Es precisamente esa alquimia de la palabra y de los números, de los sonidos, de las grafías y de las imágenes que damos a conocer aquí en un análisis semiótico de su novela Le Terrain Bouchaballe. Nuestro estudio se centra esencialmente en la descodificación de los números tres, siete y doce verificando la atracción del autor por las representaciones cabalísticas y su fe en la doctrina del Ciristo; nos confirma asimismo que las bases de la teoría cubista de Max Jacob — que caló fuerte en muchos de sus coetáneos- se estaba fraguando ya hacia 1910 , fecha en que concibió su novela.

PALABRAS CLAVES: simbolismo: número; mitología: semiótica; estética cubista.

Notre étude s'inscrit dans le cadre d'une recherche de sémiotique littéraire centrée sur le roman jacobien; elle fait suite à une analyse que nous avons réalisée au sujet du leitmotiv du «zinzolin» dans Le Terrain Bouchaballe roman de Max Jacob, publié en $1923^{2}$. C'est en étudiant la récurrence de ce terme ambivalent -couleur violette métamorphosée en étoffe solaire pour les besoins de la fiction- que nous avons pris conscience de la symbolique des nombres dans les séquences où le mot «zinzolin» joue le rôle de catalyseur. Dans la présente étude, nous avons donc choisi de retenir essentiellement les passages que privilégie le roman à partir de la présence explicite du nombre, car ils coïncident avec ceux qui véhiculent le motif de l'étoffe. Comme nous l'avons indiqué ailleurs", le mot «zinzolin», maintes fois décliné au fil du roman, se présente selon trois formes lexicales, «zinzolin, zinzoliné et zinzolinette» qui constitue une matrice. En observant de plus près la surdétermination textuelle du «zinzolin» et de ses variantes - nous recensons trente occurrences qui se regroupent en neuf séquences (c'est-à-dire trois fois trois) - nous remarquons qu'elle compose non seulement avec le nombre trois mais également avec le sept et le douze, ce qui ne peut être le fruit du hasard chez celui qui avait déjà vécu l'apparition du Christ (1909) sur le mur de sa chambre, peu avant la création des deux « Bouchaballe ».

Dès lors, nous nous proposons d'identifier les codes et les mécanismes qui nous permettent de donner une nouvelle signification du texte, celle qui se construit sur une symbolique des nombres trois, sept et douze, lesquels entrent dans un rapport d'équivalence avec le leitmotiv du «zinzolin» qui tisse le texte selon un ordre et une série de séquences savamment établies ou préétablies. Cette analyse vise à confirmer l'existence d'un imaginaire jacobien puisant dans la numérologie, l'astrologie ${ }^{4}$, le mythe, la religion ${ }^{5}$ mais aussi la fantaisie artistique où affleurent déjà les jalons de la pensée cubiste.

Nous sommes parfaitement consciente qu'une interprétation comme celle que nous proposons dans cet article peut être soumise au filtre de ce que, avec Umberto Eco

2 Voir notre article dans EPOS, XVIII (2002) Madrid, pp. 269-294.

${ }^{3}$ Ibid.

${ }^{4}$ D'une culture hétéroclite, Max Jacob s'est beaucoup intéressé aux sciences occultes et a écrit plusieurs ouvrages sur le thème de l'astrologie: "Souvenirs et astrologie» (1922:1), Le Miroir d'astrologie (1949), ouvrage posthume écrit en collaboration avec Claude Valence et Petite astrologie (1989), présentée par M. Béalu.

5 Max Jacob a lu tous les textes sacrés Évangiles, Bible... Comme l'a signalé Christine Van RoggerAndreucci (1985:184), «il cherche à travers ses différents systèmes de pensée, une ligne directrice qu'il pense trouver enfin avec le Christm. 
(1996), on a convenu d'appelé «interprétation et surinterprétation» ou, en d'autres mots, les limites des interprétations possibles entre les intentions du texte et la lecture d'un lecteur potentiel. Mais nous gageons que c'est aussi grâce à de nouveaux apports critiques que peut s'enrichir toute œuvre littéraire et c'est dans ce sens que nous souhaitons proposer cette nouvelle lecture du Terrain Bouchaballe.

Rappelons seulement pour mémoire que l'intrigue du roman repose essentiellement sur un conflit municipal, temporellement situé à la fin du XIXe siècle: l'enjeu réside dans le legs du fameux terrain, par feu André Bouchaballe, à la municipalité de sa ville qui n'est autre que la verdoyante Guichen encaissée dans une vallée à la confluence de deux rivières ${ }^{6}$; la question était de savoir si on allait y construire un théâtre (souhait du maire) ou un asile de vieillards (vœu du clergé) ou plutôt, extraire la houille que les géologues avaient signalée dans son sous-sol. Le narrateur, se faisant le chroniqueur de sa ville natale établit une impressionnante galerie de portraits qu'il ébauche avec une verve incisive digne de La Bruyère, comme il l'indique d'ailleurs ironiquement. Et le microcosme de Guichen est condamné à être enseveli par le minerai qui le souille. Opposé à toute transformation de sa charmante cité, le narrateur-chroniqueur va trouver le contrepoint de cette vision ténébreuse d'une Guichen noircie par le charbon et qui anticipe déjà l'enfer, dans l'évocation de sa chimère plus proche d'un paradis céleste?. En évoquant à plusieurs reprises les «sphères célestes" - niveau d'élévation sublime- l'auteur dévide le fil de sa chronique guichantoise avec force ironie, raillerie et sarcasme, tout en esquissant parallèlement un monde onirique où vient se greffer toute la symbolique des chiffres qu'il emprunte à des domaines variés: astrologie, mythologie, religion, liturgie, monde musical et pictural. Ce désir de mêler le merveilleux au surnaturel ne devrait pas surprendre lorsque l'on sait combien Max Jacob s'est intéressé à la numérologie et à l'astrologie ${ }^{8}$.

Dans un premier souci de repérage des nombres cités, nous analyserons les différents niveaux textuels: supraphrastique, narratif et descriptif, niveau des personnages, niveaux spatio-temporel, lexical et étymologique, phrastique, musical et pictural. Tout en suivant le cheminement de la présence numérologique et sa portée dans le roman, nous procéderons à un décodage qui tient compte de tous les niveaux textuels cités. Il en ressort que si la prégnance de l'isotopie des triades (celle du nombre trois) l'emporte, le sept et le douze n'entrent pas moins dans la dynamique d'une codification chiffrée qui force la fiction et lui donne une ampleur cosmogonique ${ }^{9}$, contrepoint du microcosme de Guichen dont le conflit est centré sur... un lopin de terre!

\footnotetext{
- Kemper, la ville natale de l’auteur signifie précisément confluence en breton; travestie en Guichen -prononcé «Guichen» ou «Guichin»-, les toponymes bretons offrant parfois des variantes phonétiques. On peut penser que Max Jacob qui parlera de Guichin dans la comédie du Terrain Bouchaballe, écrite peu avant le roman- 1910 a favorisé cette ambiguité phonétique qui a son importance, car le toponyme Guichin rimerait bien avec «zinzolin», l'étoffe si prisée par ces dames guichantoises.

7 Le mot «chimère» est maintes fois évoqué dans le roman; il renvoie à une Guichen idéalisée, apparentée à la Jérusalem céleste. Nous ne pouvons manquer de mettre en relation ces allusions avec la trilogie jacobienne de Saint Matorel, en particulier le troisième volet, Le Siège de Jérusalem, (1914).

" Max Jacob fut un temps chargé d'une chronique astrologique à l'hebdomadaire La Patrie (cf. A. Roymieux, 1994:61) et a écrit les ouvrages que l'on sait, sur le thème de l'astrologie. Jean Cocteau a pu dire de son ami Max Jacob: «astronome, astrologue, chimiste et alchimiste, mathématicien, mais de rêve, tel fut cet ami merveilleux» in Europe $36^{\circ}$ année N. $348-349$ avril-mai 1958, p. 3.

${ }^{9}$ Rappelons que la cosmogonie est une théorie scientifique ou mythique qui explique la formation de l'univers ou de certains objets célestes.
} 


\section{AU NIVEAU SUPRAPHRASTIQUE: UNE COMPOSITION TRIPARTITE}

L'architecture du roman suit un schéma précis qui révèle un souci de composition ternaire. La fiction se construit en effet sur trois volets: Livre I, Livre II et Livre III ${ }^{10}$. Le premier livre renforce à son tour cette construction triadique en proposant un titre qui présentent les trois personnages masculins les plus significatifs du roman: Le maire, le chanoine et l'architecte. Dans cette construction ternaire, on observe le même souci de gradation dans l'ordre des termes que dans le motif composé par «le zinzolin, le zinzoliné et la zinzolinette».

Par ailleurs, on notera que le chiffre sept est également codifié dans la composition romanesque, le Livre III portant le titre La femme au sept maris; de même, le nombre douze fait également partie de la facture du roman, l'auteur ayant tenu à diviser sa fiction en douze chapitres. Comme Moishe Black l'indique bien à propos au sujet du Terrain Bouchaballe, «le hasard n'a pas de place dans la composition de ce roman, la duperie, c'est de vouloir faire croire le contraire»" ${ }^{1}$.

\section{AU NIVEAU DE LA NARRATION ET DE LA DESCRIPTION}

Le «zinzolin», reçoit une place de choix dans le texte; c'est sa distribution et sa mise en valeur par la présence de ses trois variantes lexicales qui nous intéressent ici. En effet, dès l'ouverture du roman, la matrice composée par le mot clef «zinzolin» et ses deux dérivés s'impose par un soulignement ${ }^{12}$ en fin de paragraphe: «Un couturier de Paris ... a, pour des raisons plus graves qu'on ne peut croire, lancé de nouvelles étoffes de couleur violette: le zinzolin et ses compagnes, le zinzoliné, la zinzolinette, et la zinzolinette de soie...» (p. 15).

Parfois, c'est à la croisée des énoncés et de l'énonciation qu'affleure la numérologie.

Toujours en cooccurrence avec l'étoffe ou la toile dans l'extrait qui suit, le chiffre trois est doublement signifiant. Par un procédé stylistique fréquent sous la plume jacobienne - le recours au zeugma ${ }^{13}$ - le narrateur associe les trois déchirures de la toile à la phrase lacunaire qui vient exprimer l'embarras de l'interlocutrice devant la question posée $^{14}:$ «-Et Mlle Clémentine ... est ... bien!

La toile est déchirée à trois reprises, comme cette phrase (p. 203).

10) Pour faciliter notre démarche, nous soulignons les occurrences relevant des nombres trois, sept et douze dans le texte.

1 Moishe Black. University of Kakatcheswan, Canada, «Max Jacob Mystificateur, L'abbé Domnère manipulateur», $C R M J$, n. $^{\circ} 9$, p. 21.

12 On notera aussi la gradation.

${ }^{13}$ La figure du zeugma est récurrente dans le roman, (rappelons que le zeugma, est une figure de syntaxe qui consiste à réunir plusieurs membres de phrases au moyen d'un élément qu'ils ont en commun et qu'on ne répétera pas: il s'agit d'une figure elliptique).

14 Ce va et vient du mot à la chose et de la chose au mot est significatif dans le roman et dans l'œuvre de Max Jacob en général. Rappelons qu’a la fin du roman, le narrateur revient sur cette interaction par la bouche du couturier parisien qui répondit «patiemment (à Madame Assard) $1^{\%}$ que pour ses collègues et lui les mots «zinzolinette de soie» «zinzolin» manquaient aux choses ou les choses aux mots... (p. 316). 
Un second extrait fait référence à trois éléments descriptifs donnant lieu à une image marine juste avant le dialogue qui met en présence deux dames et une tierce personne (un mari absent):

C'est ainsi que la Science donne la main aux arts sous tes marronniers, ô Guichen! Le long du quai brûlant des Douanes où trois vaisseaux s'alignent, deux promeneuses discutent le feuilleton que chacune a le soir au lit:

- Si j'avais un mari comme ce...comment l'appelez-vous? ... je lui brûlerais la cervelle. -C'est un cas, ma chère, c'est un cas. (p. 20).

Quand au nombre douze, nous soulignerons la séquence où l'engouement dû à la mode du zinzolin est comparé à l'ivresse provoquée par les douze bouteilles de liqueur bues en cachette.

Dirai-je toute la vérité ? Les dames de Guichen sont moins sérieuses qu'elles le paraissent: c'est le nom du zinzolin qui a plu. Jamais on n'avait entendu un mot si joli pour désigner une étoffe! Zinzolin! Ces trois syllabes font rêver aux parfums que vous mettez sur vos cheveux le dimanche matin, mesdames! à cette liqueur dont vous avez demandé douze bouteilles à un voyageur placier et que les enfants ont bue en cachette; aux notes qui imitent la sonnerie dans certains morceaux pour piano souvent entendus avec plaisir et que vous jouez si paisiblement. C'est un mot qui fait penser aux fleurs, aux perroquets du Journal des Voyages, aux élégances de Femina et des Annales. C'est comme une dragée à liqueur. Zinzolin! Zinzolin! vous serez bientôt la joie de toute une ville et la couleur préférée des Guichantoises ${ }^{15}$ (p.16).

Au niveau de la description, nous retrouvons le nombre sept associé à la vénalité ${ }^{16}$ : il apparait en effet dans le nom de La venelle aux sept fontaines qui forme avec la rue verte le lieu mal famé de Guichen, où le narrateur situe le «théâtre d'outrages à la pudeur». On associe donc volontairement le nombre sept de cette venelle du racolage aux mythiques péchés capitaux qui, comme chacun sait, sont aussi au nombre de sept.

\section{AU NIVEAU DES PERSONNAGES}

Si l'on tient compte que Le Terrain Bouchaballe fut tout d'abord écrit pour une pièce de théâtre - comédie en trois actes (1920)- on comprend le rôle décisif des personnages et l'importance des dialogues dans le développement de l'intrigue reprise dans le roman. Affaire de mœurs et de costumes, avons-nous dit, où le zinzolin, qui fait la joie des Guichantoises, reste au centre des conversations et alimente surtout les commérages de ces dames.

$15 \mathrm{Si}$ le nombre douze est positivement connoté dans la fiction, il faut savoir qu'il ne l'était pas spécialement dans la vie de Max Jacob qui préférait tromper son monde en disant qu'il était né le 11 juillet et non pas le douze comme l'indique le registre d'état civil. L'explication, selon Frère Denis (1969:15), serait qu'il avait découvert, après avoir tiré son propre horoscope, que le 12 était un jour néfaste. Plus tard, dans La Défense de Tartuffe, il reconnaîtra pourtant «... avertissement astrologique auquel je donne trop d'importance dans ma vie: 11 juillet, espérances qui ne se réaliseront pas".

16 Il l'était déjà implicitement dans le titre du demier chapitre «La dame aux sept maris», évoqué plus haut, et qui fait allusion à la veuve Assard. 
Les trois personnages emblématiques de la ville de Guichen, le maire, le chanoine et l'architecte, sont présentés en titre du Livre 1. Contrepoint de ces trois personnages masculins, trois dames font leur apparition dès la première séquence qui, dans ce livre $I$, introduit le nom de l'étoffe «zinzolin». Il s'agit de trois Guichantoises: Mme Astic et Mme Lancret, et Mme Bidard (absente):

Mme Astic et Mme Lancret, qui vont deux fois par an chercher à Paris les nouvelles inventions de la mode, ont rapporté ces merveilles de la Capitale... - Tu verras que Mme Bidard va adopter la chose... -Elle a du goût.

Beaucoup ! mais tu sais ce qu'on en dit.

Mme Bidard à l'air d'un fauteuil...Dieu merci ce n'est pas elle qui a donné l'exemple. C'est une mère de famille et une bonne commerçante, Mme Lancret elle-même, et c'est aussi Mme Astic, une élégante véritable et une honnête femme (p. 15).

Toujours au niveau des personnages, nous vérifions une fois de plus que le choix du chiffre trois est déterminant pour indiquer le nombre d'architectes guichantois capables de réaliser la construction du théâtre de la ville sur le Terrain Bouchaballe, enjeu des plus importants dans la fiction:

Nous n'avions que trois architectes qui auraient pu présenter un devis: M. Simonnot, M. Favrel et M. Pancrasse. C'était M. Simonnot qui avait inventé la houille du terrain Bouchaballe; heurté au charbon, il ne pensait qu'à changer le pays; aucune bâtisse ne pouvait l'occuper davantage. M. Favrel, architecte diocésain, n'écoutait pas d'autres ordres que ceux de sa cliente, l'Église. Restait Pancrasse... (pp. 39-40).

Parallèlement, ce sont les trois ingénieurs belges responsables des bouleversements géologiques de Guichen, après la découverte du charbon, qui sont évoqués à l'avantdernière page du roman:

Un an après...(le mariage de Pancrasse) la houille s'élevait du royaume d'Utopie où elle torturait ses esclaves à l'empire de Guichen pour en faire des puissants. Certaines expériences de trois ingénieurs belges avaient transformé leurs doutes en certitude et leurs menaces à la paisible terre en danger. De par la civilisation! de par la science! la houille était l'assiette d'une grande cité et celle de sa fortune. Et vous, eaux vives de nos collines, futaies de nos avenues, noisetiers de nos bosquets, futaies de nos parcs, vous causiez aussi la fin de la beauté que vous aviez créée, car comme votre nombre et votre force avaient réjoui nos sensibles Guichantois ils le faisaient des nettoyeurs de schiste argileux et des boiseurs de mines (p. 317).

Enfin, nous évoquerons une dernière séquence qui concerne la gent féminine et nous paraît la plus significative: construites sur le mode de la juxtaposition, ses courtes phrases modulées par un rythme ternaire nous découvrent un cadre prolétaire celui d'un potager où trois dames vêtues de noir tricotent pour les pauvres, sous la tonnelle; leurs propos toument encore autour du zinzolin. On remarquera que, outre le nombre trois, le mot «dames» est récurrent: il apparaît trois fois, en position anaphorique et précédé d'un déictique («ces») dans deux des occurrences: 
Dans un grand potager, trois dames travaillent pour les pauvres, sous la tonnelle. Ces dames sont maigres et habillées de noir. Ces dames tricotent avec de petits crochets de fer. Le soleil est sur le jardin; l'air est frais sous la tonnelle; la rivière coule; une boule de métal pend du toit et les clématites sont comme des étoiles (p. 17).

L'anonymat des trois dames est compensé par un descriptif qui précise tout d'abord le nombre, la couleur des vêtements (celle du deuil) et leur physique, (elles sont maigres). La scène s'insère dans le jeu des oppositions qui structurent le roman: on constate, d'une part, un dynamisme dans l'action des dames (elles travaillent, tricotent) et de l'autre, le statisme des objets qui apparaissent dans le jardin potager (fixé par les verbes d'état et le verbe «pendre»). Par ailleurs, la présence de zones d'ombre et de lumière est latente dans l'évocation du noir et du soleil. Ces jeux de contrastes viennent corroborer les polarités vie versus mort et enfer versus paradis qui sous-tendent le roman ${ }^{17}$ : le mot «enfer» affleurant par attraction homonymique dans les petits crochets de fer (en fer/enfer) que manient les tricoteuses tandis que le notion de bonheur (paradis) transcende dans la lumière solaire, la métaphore florale et céleste («les clématites sont comme des étoiles») ${ }^{18}$. C'est d'ailleurs ce que confirme l'intertexte mythologique tantôt latent, tantôt explicite dans la fiction. Le décodage des signes et le glissement de sens nous permet de découvrir, dans l'anonymat des trois fileuses (vêtues de deuil), les trois Parques tissant la destinée humaine, de sorte que le jeu des correspondances se confirme dans l'opposition ombres/lumières pour établir la dualité du monde des morts et des vivants. Cette interprétation est d'autant plus probable que le narrateur fera, dans la clôture du roman, une allusion métaphorique aux Parques de la Mode en se référant aux couturiers parisiens, ces maîtres de l'Olympe de la mode dans la Capitale française ${ }^{19}$. Ici le référent mythologique est clairement exprimé: «Un de ces hommes qui sont les Parques de la Mode dans l'Olympe du Louvre, égayé par la lettre d'une cliente, se moqua d'elle, en vers au rythme d'une valse lente..." (p. 316) .

Mais l'Olympe n'est-il pas le séjour des douze grands dieux? Certes, mais il s'actualise tout autant dans le monde judéo-chrétien, autour de la figure du Christ. Car ce nombre «enivrant ${ }^{20}$ » est aussi celui des douze apôtres, des douze portes de la Jérusalem céleste $^{21}$. En effet, toute la description de la Jérusalem céleste dans l'Apocalypse est marquée par le nombre 12, celui de l'Église triomphante. Or, Guichen la verdoyante

17 Notons aussi les deux images polaires: l'astre scintillant dans le ciel et la boule de métal (que nous entendons «en fer») pendant du toit. Elles se situent sur le même axe que les précédentes et confirment la dichotomie chrétienne des deux mondes, celui d'en haut et celui d'ici-bas.

${ }_{14} \mathrm{Max}$ Jacob, a toujours interrogé le ciel et le firmament comme il s'interrogera toute sa vie sur sa mort. Ici le référent « étoile ", qui file la métaphore florale, pourrait s'inscrire a la fois dans la thématique de l'étoffe (étoile) et celle de l'astrologie associées dans le roman. Par ailleurs, on soulignera la polarité de l'astre scintillant dans le ciel et de la boule de métal (en fer?) pendant du toit. Max Jacob n'a t-il pas voulu évoquer par ces images la dichotomie des deux mondes dans la religion chrétienne?

19 Nouvelle opposition entre Guichen, petite préfecture de province et Paris, «nombril» de la France, en matière de mode.

20 Dans la fiction. les douze bouteilles bues en cachette, ne renvoient-elles à l'ivresse voire à l'extase? ou bien encore à cet état second qui permet à l'homme en quête de lui-même d'échapper aux réalités médiocres de ce bas monde? Toute la séquence se construit sur la base de synesthésies qui appellent tous les sens.

${ }_{21}$ Rappelons que dans Le siège de Jérusalem, c'est «le siège que les hommes mettent sans cesse devant Jérusalem, citadelle de la Perfection, de la Paix, du Bonheur réel» qui est évoqué. (Phrase qu 'un Ange prononce 
- pour laquelle le narrateur prend parti contre l'exploitation du charbon - est précisément comparée, avant la découverte du minerai, à un havre de paix et de bonheur, à l'instar de la Jérusalem céleste:

De par la civilisation! de par la science! la houille était l'assiette d'une grande cité et celle de sa fortune. Et vous, eaux vives de nos collines, futaies de nos avenues, noisetiers de nos bosquets, futaies de nos parcs... vous causiez la fin de la beauté que vous aviez créée (p. 317).

On notera avec quelle habileté le narrateur jacobien tisse les fils de la trame intertextuelle qui emprunte tantôt aux dieux antiques, tantôt aux sources bibliques et aux évangiles dont il est si proche ${ }^{22}$.

Il importe à présent de revenir sur le moment de la genèse de Bouchaballe et de vérifier qu'elle correspond à une période de crise mystique chez l'écrivain. En effet, la comédie et le roman ont été écrits vers 1910, même si le roman n'apparaît qu'en 1923, et le manuscrit de la comédie sera égaré puis retrouvé bien plus tard. Or, dès 1909, Max Jacob va vivre l'apparition du Christ. En 1914, nouvelle vision du Christ et en 1915 baptême. Lorsqu'il écrit son roman Le Terrain Bouchaballe, Max Jacob est donc déjà touché par la foi. Cette donnée nous paraît intéressante pour comprendre la circulation des nombres trois, sept et douze dans le roman, et leur portée symbolique en rapport avec la religion catholique qui a marqué l'œuvre jacobienne. Comme nous l'avons évoqué plus haut, c'est précisément l'isotopie des triades qui est la plus pertinente dans la fiction. Dans le contexte religieux où l'on peut situer l'auteur à l'époque de la création de Bouchaballe, on ne peut s'empêcher d'associer le nombre trois à la Sainte Trinité à laquelle l'auteur était fort attaché.

\section{AU NIVEAU SPATIO-TEMPOREL}

Nous observons également que l'intrigue est jalonnée par les trois périodes clairement définies dans le roman: la période géologique, la période oratoire et la période juridique tandis que l'espace Bouchaballe se décline lui aussi selon trois variantes qui incorporent le nom du généreux donateur:

- Le terrain Bouchaballe proprement dit, légué par feu André Bouchaballe à la municipalité de Guichen.

dans le Prologue du livre et qui, selon Raoul Auclair (1997:67) en condense tout l'argument). Signalons, d'autre part que Max Jacob y fera de nombreuses allusions au nombre douze: les douze portes, les douze perles, les douze années de siège. La référence au Zodiaque y sera tout aussi prégnante.

Ajoutons, au sujet du «douze» que, dans la bible hébraïque, la bénédiction des douze fils de Jacob (Gen. 48; 28) laisse entrevoir une certaine influence des signes du Zodiaque, et que dans le Talmud de Babylone, on y explique aussi que le nombre douze renvoie aux signes du Zodiaque, (cf. Dictionnaire critique de l'ésotérisme, sous la direction de Jean Servier, PUF, 1998).

22 Dans une conversation avec Marcel Béalu (1946:36), Max Jacob aurait commenté à son ami, à propos de l'astrologie: «Ce classement de l'humanité en douze types, pense quelle utilité il a pour un romancier» Béalu, rapporte, par ailleurs, que «longtemps l'auteur du Cabinet noir classa les lettres de ses amis en des dossiers étiquetés aux signes du Zodiaque...". 
- Le verger Bouchaballe. Il s'agit bien entendu du même terrain, mais au fil du texte, nous apprenons qu'il est planté d'arbres fruitiers et que ces arbres sont des pommiers. Sachant que la notion de péché est clairement inscrite dans le descriptif des mœurs relâchées de Guichen, l'auteur nous livre, à travers ce verger de pommiers, la confirmation de la valeur symbolique-mythologique ajoutée, celle du péché originel dans la croyance Judéo-chrétienne ${ }^{23}$.

- Le gâteau Bouchaballe. Celui-ci renvoie à un espace métaphorique qui met en scène Mme Assard et sa goinfrerie. En effet, en apprenant que Bouchaballe lui revient en héritage de son premier mari, la veuve Assard ${ }^{24}$ va, sur l'heure, commander un gâteau qu'elle ingurgite sur le champ. Par ailleurs, l'espace textuel accordé par l'auteur à ce personnage truculent -taxée d'ogresse de maris par le chanoine--, est également à tenir en compte car, nous l'avons dit, il fait l'objet de tout un chapitre, le demier du roman, sous le titre de «la dame aux sept maris».

\section{AU NIVEAU DE L'ARCHITECTURE PHRASTIQUE}

Nous signalerons également que la composition ternaire est privilégiée dans la syntaxe, le rythme, la cadence. Les séquences reprenant le mot «zinzolin» reposent alors le plus souvent sur le parallélisme syntaxique, les couplages, l'énumération et jouent sur l'homophonie; elles sont généralement au nombre de trois:

Il (le zinzolin) se marie facilement/ Il est épais et léger / Il conserve toujours son brillant et ne déteint pas au soleil.

Il sied aux brunes comme aux personnes qui prétendent au blond/ Il habille délicieusement les enfants/ Et c' est l'étoffe des personnes d'âge moyen ou des personnes âgées (pp. 15-16).

Ces trois syllabes font rêver Aux parfums que vous mettez dans vos cheveux le dimanche matin, mesdames / A cette liqueur dont vous avez demandé douze bouteilles à un voyageur placier... / Aux notes qui imitent la sonnerie dans certains morceaux de piano souvent entendus avec plaisir et que vous jouez si paisiblement... (pp. 15-16).

C'est un mot qui fait penser / -aux fleurs / -aux perroquets du Journal des Voyages / -aux élégants de Fémina et des Annales.. ${ }^{25}$

${ }^{23}$ À noter également que l'auteur a donné le patronyme de Pommier à un avoué. Il semblerait bien qu'il y ait une constante dépréciative chez Max Jacob dans le choix des patronymes désignant notaires, avocats, avoués (non seulement dans Le Terrain Bouchaballe, mais aussi dans Bourgeois de France et d'ailleurs, et dans Le Cabinet noir). La péjoration se manifeste soit par le biais de la suffixation-par exemple, suffixe en -ard, comme pour Trottard, l'avocat sanguin, (p. 168), ou Bidard l'avoué surnommé l'Alpaga, (p. 123)-. La dépréciation dans Pommier est d'un autre ordre, comme on vient de l'indiquer. Elle est confirmée dans l'apparition de cette même occurrence sous la forme du nom commun associé au péchê, voir p.122 et 123.

${ }^{24}$ Le personnage de Mme Assard est largement associé au motif du zinzolin dans le roman. Dans la demière séquence du texte, elle commandera du zinzolin pour sa robe de mariage, mais le couturier parisien lui répondra négativement et avec force ironie.

${ }^{25}$ En consultant le manuscrit du Terrain Bouchaballe à la Bibliothèque de Quimper, nous avons pu vérifier que là où apparaît le mot «zinzolin», l'auteur semble avoir voulu suivre une disposition 


\section{AU NIVEAU DE L'INTERTEXTE MUSICAL ET PICTURAL}

Finalement, nous évoquerons les dimensions musicales et picturales qui rejoignent les réseaux de significations formés par la numérologie, l'astrologie et la cosmologie religieuse.

Le rayonnement musical qui imprègne le texte ${ }^{26}$ va de l'orchestre à la musique militaire, de l'opéra aux chansons ou aux refrains populaires. Le narrateur met l'accent sur les «Merveilleuses harmonies de l'univers» dès qu'il ébauche, en quelques mots, la beauté de Guichen avec ses remparts, sa cathédrale, ses jardins, ses marronniers et son Jet (rivière) rapide qui lui aussi distille une musique toute en glouglous ${ }^{27}$. Ce même narrateur s'insurge contre ceux qui ne comprennent rien à l'harmonie des sphères: «S'agit-il oui ou non d'exprimer l'harmonie des sphères? Oseriez-vous prétendre que la sphère des trombones est inférieure à la sphère piano?» (p. 24).

Par ailleurs, la question Bouchaballe, reliée à la question du théâtre, est aussi une affaire de mœurs et de costumes où - ajoutera le narrateur - «la musique d'orchestre est principalement en question ${ }^{28}$. En effet, dès la partie liminaire du roman, c'est à travers les notes musicales que s'établissent les correspondances entre le sonore zinzolin et l'harmonie des pièces pianistiques. Si le narrateur met en évidence le nombre trois à propos de la division syllabique du mot clef, il n'en évoque pas moins le nombre sept par allusion aux sept notes de la gamme: «Zinzolin! Ces trois syllabes font rêver aux parfums... aux notes qui imitent la sonnerie dans certains morceaux pour piano souvent entendus avec plaisir et que vous jouez si paisiblement...» (p. 16).

Puis, la rêverie musicale va céder le pas à un rythme harmonieux, dans une séquence où le zinzolin ou plutôt la rieuse et affective zinzolinette —qui dans une lecture rapide passerait pour un détail vestimentaire anodin- établit un contrepoint à la fois élégant et pictural: «Le décor chancelle. Guettant le cortège, un ruban en zinzolinette au cou, la caissière du café Prosper bat la mesure discrètement avec ses clefs (...) Une paysanne dispose les chaises pour la musique du soir. Et puis c'est tout.» (p. 25).

Dans ce portrait tout en mouvement, le jeu homophonique permet au narrateur d'évoquer le thème musical, grâce au glissement de sens du mot «clefs» qui évoque à la fois, la serrure et par association métonymique l'ouverture, et partant le sésame (et le zinzolin) et la clef de la gamme des sept notes. Le lecteur voit ainsi apparaître en filigrane, la clef de sol qui associe la lexie solaire ${ }^{29}$ du zinzolin — par l'entremise de la zinzolinette-, au signe musical. En l'absence d'une allusion chromatique précise, en ce qui concerne la zinzolinette, c'est la forme qui est privilégiée: le ruban noué autour du

typographique particulière, pour mettre en valeur le terme, de sorte que "zinzolin" apparaît en position anaphorique, en finale, après un point, entre deux exclamations... Par ailleurs, il apparaît que Max jacob a également pris plaisir à tracer le graphe initial $-\mathrm{Z}-$ en lui donnant un léger prolongement en forme d'arrondi dans chacune des séquences. Cependant la séquence citée est écrite d'un seul bloc. la disposition que nous lui donnons ici n'a d'autre objectif que de souligner les structures parallèles et le jeu des anaphores par groupe de trois.

${ }^{2 h}$ Et pourrait constitué à lui seul un sujet de recherche.

${ }^{27}$ La rivière est aussi associée à la musique par le nom de la patronne des musiciens: «La rivière se courbe quand la rue Sainte-Cécile, qui n'a pas de maisons, divise le Petit-Quai en deux parties» (p. 18).

${ }^{28}$ P. 28.

${ }^{29}$ Par ailleurs, le thème astral est également évoqué à travers le nombre sept qui est celui des planètes. 
cou de la caissière évoque alors la figure géométrique du cercle, référent harmonieux par excellence ${ }^{30}$.

Au fur et à mesure que la fiction prend forme, la musique se confond avec les instruments et la rêverie cède le pas à une musique jouée par un instrument musical précis, le triangle ${ }^{31}$. Nous citerons les séquences, très rapprochées dans le roman, où l'instrument privilégié compose un espace musical sur une base tri-angulaire: «C'est ennuyant, monsieur Mouzot, dit Curot, je crois que je ne sais pas bien ma partie de triangle [...] Cependant, Curot a les castagnettes et Mouzot en tête joue du triangle» (p. 24).

La dernière allusion au triangle appartient exclusivement au domaine pictural: c'est l'image d'un dernier rayon de lumière (artificielle) contrepoint nocturne de la lumière solaire (diurne) qui irradie la ville de Guichen à travers l'étoffe du zinzolin: «La table de l'hôtel Prosper n'est plus qu'un dernier triangle de gaz sous les marronniers» (p. 25).

Le choix de cet instrument musical n'est pas innocent. La polysémie du mot triangle, permet au narrateur de passer du son à la forme, de toucher au domaine à la fois musical et pictural. En évoquant le polygone, c'est la facette cubiste -celle de la représentation géométrique de l'objet dont il a souvent fait l'éloge- qu'jl nous dévoile. Le triangle (trois côtés, trois angles) sert alors de catalyseur au narrateur pour construire un ordre cosmique idéalement établi dans une nouvelle harmonie qui emprunte aux astres, à la musique et à la forme ${ }^{32}$...

\section{AuX NIVEAUX ÉTYMOLOGIQUE, LEXICAL ET GRAPHIQUE}

Nous l'avons dit plus haut, le choix d'une numérologie singulière et récurrente, dans le roman nous a amenée à établir un rapport de correspondance avec le nom du zinzolin, nom que l'auteur métamorphose en étoffe solaire («étoffe tissé avec le soleil», p. 26) dans sa fiction. Le narrateur, créateur d'équivoques ${ }^{33}$, va volontairement composer avec une étymologie fantaisiste qui fait intervenir trois codes linguistiques différents, l'anglais, l'espagnol et le français. Une fois le mot décomposé, le narrateur «se gargarise ${ }^{34}$ de chacune des lexies (sun/sol/sol-eil) en les soulignant par trois occurrences (si nous tenons compte de l'apocope de soleil en sol):

- Il y a, dit le professeur de cinquième qui ose parler quand la rivière fait du vacarme, dans zinzolin, la racine sol, le préfixe sun et le suffixe lin; sun, sol, lin, étoffe tissée avec le soleil, sun avec ! C'est très beau... (p. 26).

\footnotetext{
30) Jean-Marc Pontier remarque que «C'est l'image du cercle qui prédomine dans l'œuvre de Max Jacob. Les cercles, sphères ou disques développent une esthétique de la rondeur», dans «Max Jacob du poème au dessin», Thèse de doctorat, Université d'Orléans, 1995, p. 112.

31 Précisons qu'en termes d'harmonie, la sonorité du triangle est caractérisée par le grand nombre de sons harmoniques qui accompagnent le son fondamental. Cf. Marc Honegger (2002) Dictionnaire du musicien. Les notions fondamentales, Larousse.

32 «Je crois que tout est polygone [...] N'oublie pas que toute forme doit pouvoir se ramener à la géométrie et que la géométrie est une aide forte», cité par Roger Toulouse (1987:39), in «Max Jacob, peintre».

33. Le peintre Roger Toulouse (1985:88) note également que Max Jacob aimait égarer son lecteur.

${ }_{34}$ Est-il nécessaire de rappeler l'un des vers les plus cités de Max Jacob: «Aimer les mots- Aimer un mot. Le répéter, s'en gargariser. Comme un peintre aime une ligne, une forme, une couleur (très important)», in Conseils à un jeune poète (1945:35).
} 
En remontant aux sources du mot zinzolin, on apprend que ce terme signifie «couleur violette que l'on obtient du sésame» et qu'il procède de l'arabe d'Espagne, «djoujoulan», ce qui signifie: "semence de sésame». Terme d'une fréquence rare dans la langue courante, mais surdéterminé dans le roman, le mot devient alors porteur de significations multiples dans un champ ouvert aux connotations qui joignent le fantastique au merveilleux ${ }^{35}$. Car le sésame, c'est avant tout une porte qui s'ouvre sur un monde fabuleux nous découvrant un espace magique, surnaturel. L'ailleurs que dévoile le zinzolin s'apparente alors à l'Eden, contrepoint des zones d'ombre, celles de la menace déjà évoquée du charbon noir guichantois et associées à l'image de l'abîme, de l'enfer, dès lors que la paisible et rieuse Guichen est menacée par le progrès. Dès le début du roman, le narrateur présente cette lexie trisyllabique en mettant précisément en relief sa composition ternaire - «ces trois syllabes»- et en créant des synesthésies qui allient odeur, parfums et ivresse dans l'évocation des douze bouteilles bues en cachette ${ }^{36}$.

C'est donc dans l'étymologie de ce terme d'origine exotique et triplement solaire (dans la fiction) que nous trouvons les connotations à la fois magiques et astrologiques rattachées à l'étoffe prisée par les dames de Guichen ${ }^{37}$.

Le décryptage abordé au niveau étymologique va se poursuivre au niveau graphémique, toujours dans le rapport associé au «zinzolin» qui, nous l'avons dit, fait l'objet d'un soulignement pondéré dans le roman. Grâce à sa récursivité et sa polysémie, cet élément polyphonique dynamise le texte; qu'il fasse cavalier seul, qu'il soit couplé (Zinzolin! Zinzolin!) ou qu'il apparaisse avec son cortège de dérivés (zinzoliné, zinzolinette, zinzolinette de soie), le mot porte presque toujours la majuscule grâce à une distribution précise et une ponctuation privilégiées au niveau stylistique. Forte de son «Z» initial, la lexie redouble cette lettre, ce qui concourt à renforcer la prégnance du graphème dont le tracé géométrique, composé de trois traits, semble parcourir la page du texte, telle une signature cryptée. En observant de plus près, on vérifie que ce «Z», ultime lettre de notre alphabet, se situe ici en position initiale (et médiane) dans le mot clef «zinzolin» ce qui corrobore les polarités textuelles s'actualisant une fois de plus au niveau graphémique. Mais le graphème $\mathrm{Z}$ que Max Jacob prend tant de plaisir à dessiner dans son manuscrit ${ }^{38}{ }^{\prime}$ 'est-il pas également présent dans l'initiale du mot Zodiaque, toujours porteur d'une majuscule dans le texte, et dont la récurrence est également notable? Cette surdétermination graphique reste donc à expliquer: nous venons de voir que graphie et lexie participent de la numérologie, dès lors que les chiffres trois, sept, douze sont évoqués dans des séquences où le mot «zinzolin» est l'élément codé; par ailleurs, nous avons vu que le « $Z$ » du Zinzolin fait figure de sésame, d'ouverture vers une

35 Christine Van Rogger-Andreucci, (1985: 85) qui a étudié la lecture des évangiles chez Max Jacob, signale: «La science symbolique est d'abord celle du nombre et du nom. On comprend combien a pu le séduire l'idée de la valeur sacrée du verbe, de la puissance du nom telle qu'il la trouve chez les Kabbalistes [...] Mais si les kabbalistes ont recours à un système d'interprétation complexe, Max Jacob utilise plus simplement souvent la décomposition étymologique...».

*6 Ivresse dans le sens de volupté et «en cachette» en relation à la faute, à l'interdit, au péché. Voir la citation complète plus haut.

37 «Je crois fermement à l'astrologie, m’écrivait Max Jacob, le 18 mai 1940, mais rarement aux astrologues», cité par Marcel Béalu (1989:6) dans la présentation de Petite astrologie de Max Jacob; livre qui a été transcrit par M. Béalu en 1939, sous la dictée de M. Jacob qui ne l'a jamais relu.

38 Voir plus haut. 
imagination qui donne libre cours à l'ivresse, aux parfums, aux fruits défendus ... Parallèlement, le «Z» du Zodiaque s'ouvre sur un univers de promesses, d'espoir qu'ébauchent les lignes du firmament et que le narrateur transpose géométriquement sur le papier. Or, il s'avère que graphie et nombre se confondent dans le tracé du Z que l'auteur nous induit à lire comme un 7 sept à l'envers ${ }^{39}$. Claudel a jadis attiré l'attention sur le rôle des formes graphiques dans la rêverie des mots. Max Jacob nous offre, dans Le Terrain Bouchaballe, un exemple de cette capacité évocatrice d'un graphème, en nous invitant à retoumer la lettre et la lire à (l'en-vers) pour mieux décrypter l'intention du narrateur ${ }^{40}$.

Cette interprétation serait peu consistante si elle n'était étayée par le texte luimême. En effet, la fin du roman nous donne la clé, appelons-la "sésame», pour déchiffrer cette énigme. C'est le couturier parisien - un de ces Parques de la mode dans l'Olympe-qui nous met sur la piste en parlant de lettre en vers (à l'envers) dans sa missive ironique adressée à Mme Assard. Relisons l'extrait déjà cité plus haut:

Un de ses hommes qui sont les Parques de la Mode dans l'Olympe du Louvre, égayé par la lettre d'une cliente, se moqua d'elle, en vers au rythme d'une valse lente. Puis il répondit patiemment 1 " que pour ses collègues et lui les mots «zinzolinette de soie» "zinzolin " manquaient aux choses ou les choses aux mots... (p. 316).

Rappelons que Mme Assard, est «la dame aux sept maris». L'auteur, en forçant sur l'attraction paronymique, associe le nom propre Assard au nom commun «hasard»41. C'est ainsi que Mme Assard acceptera de se faire tirer les cartes pour trouver un mari à son goût, c'est-à-dire, riche et de noble origine ${ }^{42}$ et que le hasard ou la rencontre fortuite favorisera l'alliance avec Pancrasse qui sera son dernier mari ${ }^{43}$. Rappelons que l'auteur du Cornet à dés tirait souvent les cartes et établissait des horoscopes, ce même horoscope qui renvoit au Zodiaque et partant au nombre douze, celui des douze signes astrologiques. Qu'il soit associé aux astres, à l'Évangile, au Christ, où aux Dieux de l'Olympe, le douze se présente comme le nombre solaire, à l'instar du zinzolin transformé en étoffe, tissée par le soleil sous la plume du narrateur guichantois.

${ }^{39}$ On pourrait évoquer également le nom de Babylone qui provient du nom hébreu, Babel et signifie la Porte de Dieu en langue sémitique. Babylone est connue pour ses jardins suspendus, une des SEPT merveilles du monde. La relation entre Guichen et Babylone est établie par l'Abbé Davant qui compare la petite ville bretonne à la ville mythique (p. 42 ).

4) René Guy Cadou (1969:12) a évoqué le goût de Max Jacob pour écrire à l'envers: «Je me souviens de ses lettres». Cette Madame de Sévigné en pantalon de velours et gros sabots écrivait à l'envers des propos qui auraient fait la fortune des gazettes, d'une sagesse suffisante à la gloire d'un homme, dans Pour en revenir à Max Jacob, José Millas Martin, éditeur, Paris.

4) Faut-il rappeler que l'étymologie du mot «hasard» renvoie au dé et au jeu de dés et que Max Jacob est l'auteur du recueil de poèmes Le cornet à dés.

(Cf. Le Robert: hasart, 1155, au sens I, 1; hazard, XVe, au sens II; arabe (") az-zahr «dé, jeu de dé», par l'intermédiaire de l'esp. azar, l'orig. du mot arabe est controversée, soit de yasara «jouer aux dés», soit de zahr "fleur»; cf. esp. azahar «fleur d'oranger», les dés ayant porté une fleur sur une face).

${ }^{42}$ Ce n'est pas un hasard si le prénom de Mme Assard, est Eugénie dont l'étymologie (đu grec, «de noble race» expliquerait son désir d'alliance aristocratique.

${ }^{43}$ Pancrasse qui n'est ni riche ni noble se vantera d'avoir un profil bourbon (p. 210), et son cousin, le chanoine, commentera à son sujet: «[...] ce de fils naturel d'une cuisinière a l'intelligence aristocratique» (p. 264). 
Chez Max Jacob, le romancier, le poète, l'astrologue ${ }^{44}$, le musicien et le peintredessinateur sont un seul et même créateur aux multiples facettes. Sachant que la plupart de ses manuscrits et ses livres portent une signature picturale qui forme une osmose avec l'ouvrage littéraire, pour son roman Bouchaballe cette signature graphique et picturale ne pouvait manquer. La consultation du manuscrit autographe du Terrain Bouchaballe (Ms Bibliothèque de Quimper) nous a permis de vérifier que Max Jacob nous a légué un dessin symbolique, tout à fait interprétable dans la ligne sémiotique qui construit sa fiction ${ }^{45}$ : on y découvre des tracés géométriques, des lignes reliant des constellations dans le ciel, et un astre: cet astre, évidemment, c'est le soleil. Le mot soleil s'inscrit dans un cercle où trône un personnage royal, tel un démiurge. Le dessin nous confirme que l'ouvrage se forge sous le signe solaire qui, comme nous l'avons démontré, transcende toute la fiction. Il vient alors étayer notre hypothèse de départ, celle d'une correspondance entre leitmotiv du zinzolin, l'étoffe solaire et les isotopies des nombres codés ${ }^{46}$.

Sachant que le microcosme de Guichen est voué à la disparition, le narrateur arpenteur aura délibérément choisi le trois, le sept et le douze comme base géométrique pour réordonner le cosmos et le concevoir à l'aune d'une dimension à la fois numérique, astrologique, mythologique et religieuse. Auteur et narrateur omniscient se confondent dans ce roman où Max Jacob parle de la résurrection de sa ville natale qu'il associe, le temps de quelques images, à la Jérusalem céleste (celle de $\mathrm{Matore}^{47}$ ) où les mots paix, bonheur et paradis devaient être inscrits pour l'éternité.

\section{BIBLIOGRAPHIE}

JACOB, Max (1964): Le Terrain Bouchaballe, Paris, Gallimard.

JACOB, Max (1909): Saint Matorel, roman suivi des CEuvres burlesques et mystique de Frère Matorel mort au couvent de Barcelone et du Siège de Jérusalem, drame céleste, 3 vol., édition originale avec illustrations de Picasso et André Derain.

JACOB, Max (1920): «Plus d'astrologie» (poème), Action, mars 1920, p. 26.

JАСОВ, Max (1922): «Souvenirs et astrologie», Les Nouvelles Littéraires, n. ${ }^{\circ} 586$, samedi 19 août 1922, p. 1.

JACOB, Max en collaboration avec Claude Valence (1949): Le Miroir d'astrologie, ouvrage posthume, Paris, Gallimard.

JACOB, Max (1960): Le Laboratoire central: poèmes. Préface d'Yvon Belaval, Paris, Gallimard. JACOB, Max (1966): onseils à un jeune poète, Paris, Gallimard.

JACOB, Max (1967): Le Cornet à dés, Paris, Gallimard.

JACOB, Max (1994): L'amitié: lettres à Charles Goldblatt, édition établie et présentée par André Roumieux, Le Castor Astral.

44 Dans Le Laboratoire Central (1980:87) Max Jacob affirmera avoir renoncé à l'astrologie, incompatible avec la religion. C'est dans le poème intitulé «plus d'astrologie», qu'il nous donnera ces deux vers:

«Et l'œil du Christ ayant les clefs de mon logis/ $\mathrm{A}$ jamais je renonce à toi, l'astrologie».

45. Dans une lettre inédite datée du 3 juin 1922, Max Jacob dira à son ami Cocteau: «Je finis un gros roman Le Terrain Bouchaballe: je ne me suis jamais senti si cubiste depuis que je fais 'du réel'».

4h Nous joignons en annexe le cliché du dessin, aimablement cédé par la Bibliothèque de Quimper.

${ }^{47}$ Cf. l'article de Raoul Auclair (1997:67-70), «Max Jacob qui fut Saint Matorel». 
JACOB, Max; CoCTEAU, Jean (2000): Correspondance 19/7-1944, texte établi et présenté par Anne Kimball, Paris, Édition Paris-Méditerranée.

AuClaIR, Raoul (1997): «Max Jacob qui fut Saint Matorel», Max Jacob et la création, Jean Michel Place, éd. pp. 67-70.

BÉalu, Marcel (1946): Dernier visage de Max Jacob 1937-1944, Editeur Pierre Fanlac.

BÉALU, Marcel (1989): présentation de Petite astrologie de Max Jacob, Le bibliophile Rémois. Livre transcrit par M. Béalu en 1929 sous la dictée de M. Jacob.

BraCH, Jean-Pierte (1994): La symbolique des nombres, Paris, PUF, Que sais-je?

CADOU, René Guy (1969): Pour en revenir à Max Jacob, José Millas Martin, éditeur, Paris.

DARRY, A. R. (1966): La philosophie des nombres, Paris, Les éditions des Champs-Élysées.

ECO, Umberto (1996): Interprétation et surinterprétation, Paris, PUF coll. Formes sémiotiques.

Durand Guizıou, Marie-Claire: «À propos d'une dédicace poétique de Max Jacob à André Salmon», Philologica Canariensia n. ${ }^{\circ}$ 8-9 (2002-2003), Las Palmas, pp. 76-85.

Durand Guizıou, Marie-Claire: “ Le zinzolin, le zinzoliné et la zinzolinette,étude du motif de l'étoffe dans Le Terrain Bouchaballe de Max Jacob, EPOS, XVII (2002), Madrid, pp. 266-294.

Durand Guiziou, Marie-Claire (2004): Sous le pont Max Jacob coule le Jet et...», Isla abierta. Estudios franceses en memoria de Alejandro Cioranescu, La Laguna, Servicio de Publicaciones de la Universidad de La Laguna, 3 vols., pp. 483-496 vol. I.

FRÈre DENIS, O. R. Bénédictin (1969): «Itinéraire spirituel avec Max Jacob», in Pour en revenir a Max Jacob, Paris, José Millas-Martin éditeur.

Gнука C. Matila (1931-1935): Le nombre d'or: rites et rythmes pythagoriciens dans le développement de la civilisation occidentale, Paris, Édition de la Nouvelle revue française, 2 volumes.

HONEGER, Marc (2002): Dictionnaire du musicien. Les notions fondamentales, Paris, Larousse.

IFRAH, Georges (1981): Histoire universelle des chiffres, Paris, Seghers.

JAMIE, James (1997): La musique des sphères: musique, science et ordre naturel de l'univers, édition du Rocher - J.-P. Bertrand.

SERVIER, Jean, sous la dir. de (1998): Dictionnaire critique de l'ésotérisme, Paris, P.U.F.

TEMPLE, Éric (1952): La magie des nombres, Paris, Payot.

TOULOUSE, Roger (1987): «Max Jacob, peintre», Qui (ne) connaît (pas) Max jacob. Max Jacob et la Création, Université du Troisième Âge de Vannes, Institut Culturel de Bretagne, p. 39.

VAN RogGer-ANDREuCCl, Christine (1985): «La lecture des évangiles: une herméneutique ésotérique», Centre de Recherche Max Jacob, n. ${ }^{\circ}$ 7, Publications de l'Université de SaintÉtienne. 
ANNEXE

Cliché des dessins représentant trois cercles dont le plus important contient l'astre solaire. Max Jacob les a fixés à la page 26 verso (haut de la page, à gauche) du manuscrit autographe du Terrain Bouchaballe. Le cliché nous a aimablement été cédé par la Bibliothèque municipale de Quimper.

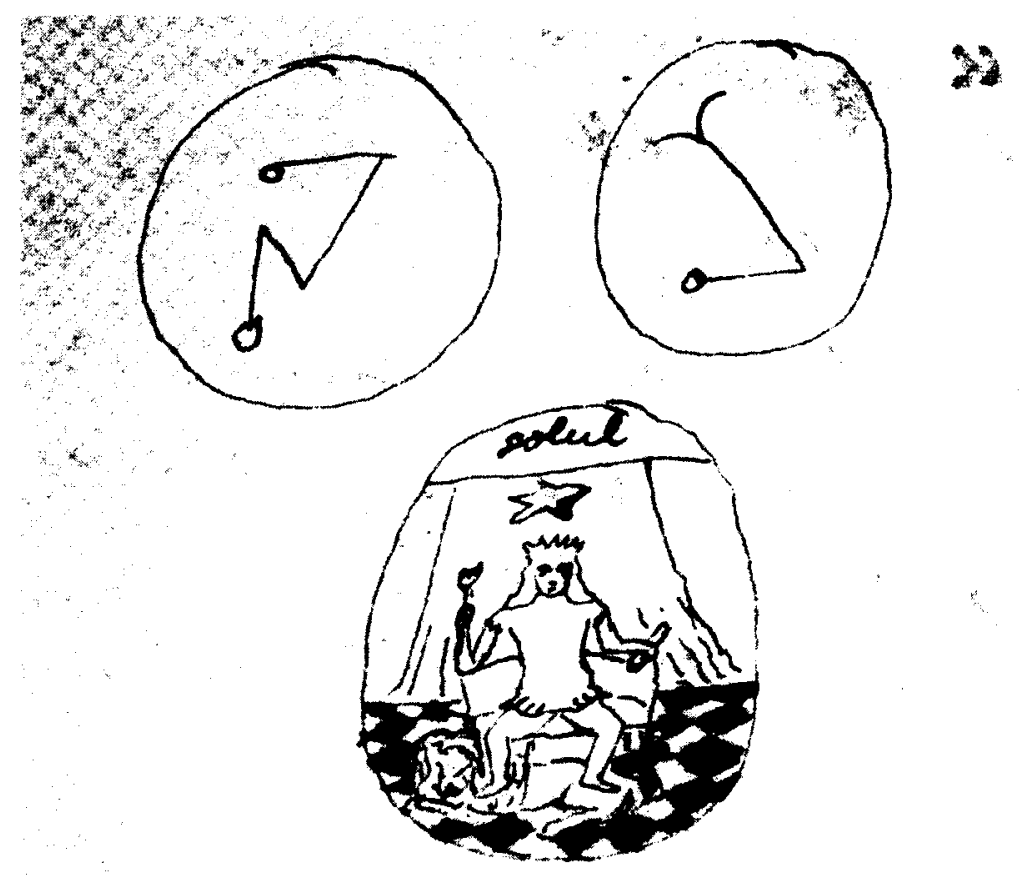

